\title{
Enhancement of Proton Transport in an Oriented Polypeptide Thin Film
}

\author{
Yuki Nagao, ${ }^{*} \dagger$ Jun Matsui, ${ }^{\ddagger} \diamond$ Takashi Abe, ${ }^{\S}$ Hirotsugu Hiramatsu,, Hitoshi Yamamoto, ${ }^{\perp}$ \\ Tokuji Miyashita, ${ }^{\ddagger}$ Noriko Sata, ${ }^{\#}$ and Hiroo Yugami ${ }^{\nabla}$ \\ ${ }^{\dagger}$ School of Materials Science, Japan Advanced Institute of Science and Technology, 1-1 Asahidai, Nomi, Ishikawa 923-1292, Japan \\ ${ }^{\ddagger}$ Institute of Multidisciplinary Research for Advanced Materials (IMRAM), Tohoku University, 2-1-1 Katahira, Sendai 980-8577, \\ Japan \\ ${ }^{\S}$ Graduate School of Science and Technology, Niigata University, 8050 Ikarashi 2-no-cho, Nishi-ku, Niigata 950-2181, Japan \\ "Graduate School of Pharmaceutical Sciences, Tohoku University, 6-3 Aoba Aramaki, Aoba-ku, Sendai 980-8578, Japan \\ ${ }^{\perp}$ Department of Safety and Hygiene, Osaka University, 1-1 Yamadaoka Suita, Osaka 565-0871, Japan \\ "\#nstitute of Technical Thermodynamics, German Aerospace Center (DLR), 38-40 Pfaffenwaldring, 70569 Stuttgart, Germany

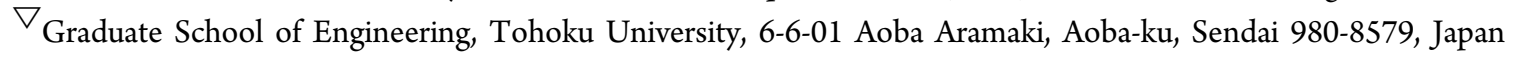

Supporting Information

ABSTRACT: Proton transport properties of a partially protonated poly(aspartic acid)/sodium polyaspartate (P-Asp) were investigated. A remarkable enhancement of proton conductivity has been achieved in the thin film. Proton conductivity of $60-\mathrm{nm}$-thick thin film prepared on $\mathrm{MgO}(100)$ substrate was $3.4 \times 10^{-3} \mathrm{~S} \mathrm{~cm}^{-1}$ at $298 \mathrm{~K}$. The electrical conductivity of the oriented thin film was 1 order of magnitude higher than the bulk specimen, and the activation energies for the proton conductivity were $0.34 \mathrm{eV}$ for the oriented thin film and $0.65 \mathrm{eV}$ for the pelletized sample, respectively. This enhancement of the proton transport is attributable to the highly oriented structure on $\mathrm{MgO}(100)$ substrate. This result proposes great potential for a new strategy to produce a highly proton-conductive material using the concept of an oriented thin film structure without strong acid groups.

\section{INTRODUCTION}

Polymer electrolyte membrane fuel cells (PEFCs) are ideal power sources having high efficiency and power-to-weight ratios over other portable power devices. Remarkable progress is noted in the research and development of PEFCs in recent decades. Despite the significant numbers of polymer electrolyte materials that have been developed and studied so far, commercialization of PEFCs needs a breakthrough in the development of electrolytes of high proton conductivity, material stability as well as low cost. In those proton conducting polymers, a decrease in proton conductivity is a trade-off between performance and membrane stability. One fundamental approach to create highly proton-conductive material is chemical modification. Usually, sulfonic acid groups are used as a proton conductive group because of their excellent property to achieve high proton conductivity. ${ }^{1-6}$ However, the high acidity of sulfonic groups restricts the polymer backbone to fluoro or aromatic groups, which requires high production costs. Therefore, a new strategy for low cost production of highly proton-conductive materials has been sought for some time.

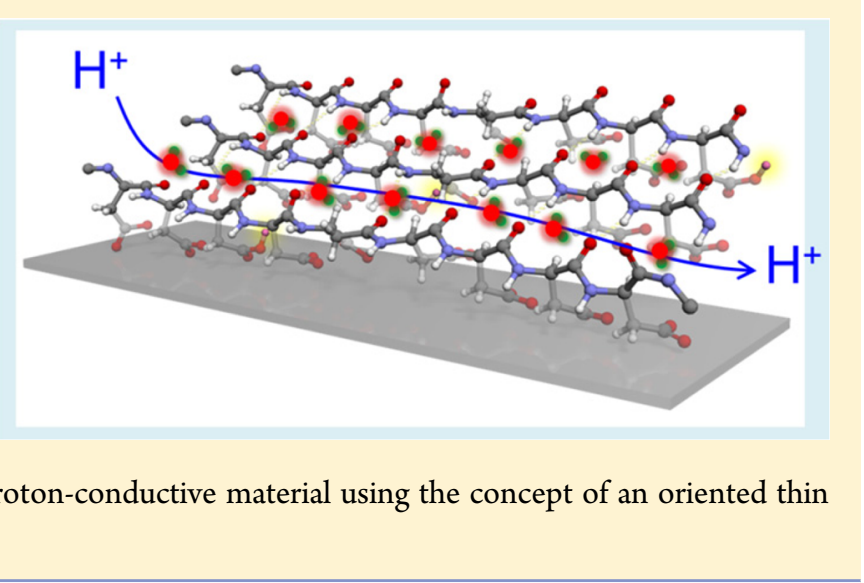

In a Nafion membrane, it is well known that protons are transported through nanochannels made of sulfonic acid groups, which has been intensively discussed by many researchers. $^{7-10}$ The nanochannels are created by phase separation with the amphiphilic character of Nafion. The proton transport property of Nafion is excellent when the PEFC is operated below $80^{\circ} \mathrm{C}$. It is, however, highly imperative to improve both the proton transport property and the stability at higher temperatures, which is limited within the concept of using microphase separation. Our group found that the proton conductivity of the Nafion thin film (thickness $\approx 400 \mathrm{~nm}$ ) was extremely decreased compared to that of the commercial membrane (thickness $\approx 200 \mu \mathrm{m}$ ). ${ }^{11,12}$ In the thin film, the thin film was fabricated on a flat substrate, and the conductivity measurement has been performed parallel to the film surface. We have found that the sulfonic acid groups are highly oriented to form dimer or trimer by hydrogen bonds in between. It is

Received: January 30, 2013

Revised: $\quad$ May 11, 2013

Published: May 30, 2013 
Scheme 1. Synthesis of Partially Protonated Poly(aspartic acid)/Sodium Polyaspartate (P-Asp)

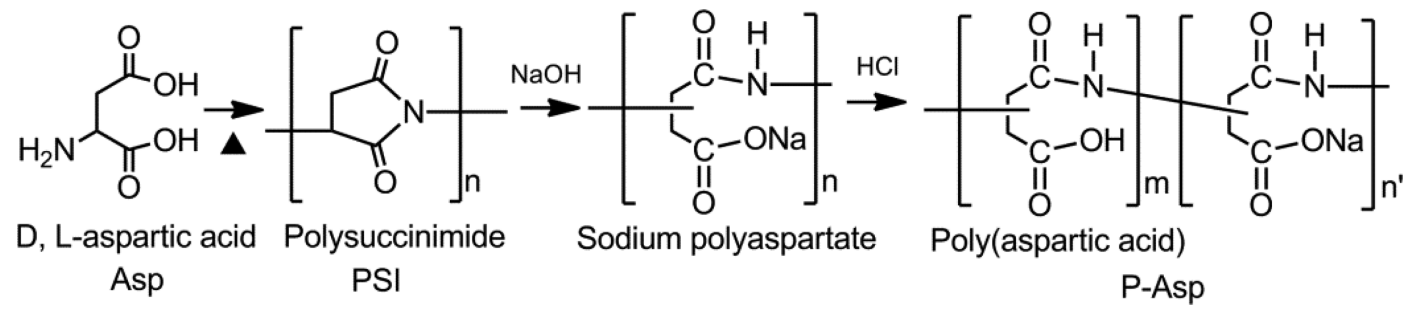

suggested that the oriented structure blocks the ion conduction path parallel to the substrate surface by the isolated sulfonic acid group phase and the ion conductivity is dramatically decreased. Our results demonstrated that the molecular orientation of the sulfonic acid groups may significantly affect the proton transport property. Therefore, the microphase separation as well as the proton conductive group orientation will be the key properties to improve proton conductivity.

Our group has been studying to find a new and different concept from Nafion and to synthesize proton conductors using poly(aspartic acid). Poly(aspartic acid) has free carboxylic acid groups at the side chains, and the protons at these groups are mobile for proton conduction. It is well acknowledged that amino acid polymers take several hierarchical structures, such as $\alpha$-helix or $\beta$-sheets via hydrogen bonding between the amino acids. We, therefore, speculated that a high proton conduction path will be achieved when an ordered proton conductive channel is formed by such hierarchical structures. It is quite different from the case of Nafion membrane, in which microphase separation leads to the high proton conductivity. We have recently reported a significantly high proton conductivity in the thin films of poly(aspartic acid) fabricated on fused silica substrates. ${ }^{13}$ The proton conductivity of the thin film was one order of magnitude higher than that of the pelletized specimen. It is suggested that the origin for the proton conductivity enhancement was due to the structural effects, as we speculated, such as molecular orientation. However, further structural analyses are required to confirm the origin of the conductivity enhancement.

In this study, we investigated the proton transport properties of a partially protonated poly(aspartic acid)/sodium polyaspartate (P-Asp) thin film prepared on $\mathrm{MgO}(100)$ substrate by spin coating and the relationship between the molecular orientation and proton transport property was investigated using the amino acid polymer. Infrared spectroscopy, i.e., infrared reflection absorption spectroscopy (RAS) and $p$ polarized multiple-angle incidence resolution ( $p$-MAIR) technique, ${ }^{14-17}$ has been performed using $\mathrm{MgO}$ substrate, which is transparent in the correspondent IR region to determine the thin film structure and the mechanism of proton conductivity enhancement discussed in this work. We found a highly oriented structure of the peptide groups in the thin film on $\mathrm{MgO}(100)$ substrate, induced the enhancement effect to the proton conduction.

\section{EXPERIMENTAL SECTION}

P-Asp was synthesized according to Scheme $1 .{ }^{13}$ D,L-Aspartic acid, $o$ phosphoric acid, methanol, sodium hydroxide, and $\mathrm{HCl}$ were purchased from Wako Pure Chemical Industries Ltd. Partially protonated poly(aspartic acid)/sodium polyaspartate (P-Asp) was synthesized as follows (Scheme 1). A 1-g portion of D,L-aspartic acid and a $600-\mu \mathrm{L}$ portion $85 \% o$-phosphoric acid of were added to a 100 $\mathrm{mL}$ round-bottom flask and mixed for $10 \mathrm{~min}$ under $\mathrm{Ar}$ atmosphere.
The mixture was heated at $170{ }^{\circ} \mathrm{C}$ for $1 \mathrm{~h}$. The product was washed with methanol and water. The residue was washed with methanol and dried under reduced pressure. A 0.7-g portion of the dried sample and a 0.3 -g solution of sodium hydroxide with $20 \mathrm{~mL}$ of deionized water were added to a $200-\mathrm{mL}$ beaker with stirring for $20 \mathrm{~min}$. After the reaction, the solution was adjusted to about $\mathrm{pH} 6$ by the addition of $35 \%$ aqueous $\mathrm{HCl}$ into the solution. A white precipitate was obtained by adding $100 \mathrm{~mL}$ of methanol. The sample was washed with methanol and was separated from the supernatant, in which the centrifuge technique was used in the washing and separation procedures. The obtained sample was dried under reduced pressure.

The sample was characterized via ${ }^{1} \mathrm{H}$ NMR and FT-IR measurements. ${ }^{13}$ The average degree of polymerization was ca. 50, which was determined by comparing the ratio of the peak area derived from the proton of $-\mathrm{CH}_{2}$ at the amino end groups $(\delta=2.95-3.25 \mathrm{ppm})$ to that of the intrachain $-\mathrm{CH}_{2}$ and $-\mathrm{CH}_{2}$ at the carboxyl end groups. The degree of protonation was found to be $6 \%$ using the titrimetric method. The P-Asp thin film was prepared by spin coating using a spincoater (ACT-200; Active) on a mirror-polished $\mathrm{MgO}(100)$ substrate. $\mathrm{MgO}(100)$ substrate was purchased from Neotron Co., Ltd. The $\mathrm{MgO}(100)$ substrate size was $15 \times 15 \times 0.5 \mathrm{~mm}^{3}$. Before spincoating, the substrate was irradiated by UV light for $1 \mathrm{~h}$ to clean the surface. The thickness and surface roughness were determined using a contact stylus profiler (P-10; KLA-Tencor Corp.). The thicknesses of the thin films were $20-60 \mathrm{~nm}$. The roughness measurement secured us its smoothness, which was sufficient for impedance analysis.

Impedance measurements of the pelletized sample and thin films were conducted at a relative humidity (RH) of $40-70 \%$ using an Impedance/Gain-Phase analyzer (SI1260; Solartron Analytical) and a Dielectric Interface system (1296; Solartron Analytical). The RH and temperature were controlled using a humidity-controlled and temperature-controlled chamber (SH-221; Espec Corp.). For impedance measurements of the pelletized sample, the sample was processed into pellets of $2.5 \mathrm{~mm} \phi$ under a pressure of about $1 \mathrm{GPa}$ and porous gold paint (SILBEST No. 8560; Tokuriki Chemical Research) was used for electrodes. For impedance measurements of the thin films, the electrode configuration was selected to obtain measurements of the current flow in the plane parallel to the substrate surface. Typical complex impedance plots of the P-Asp thin film are shown in Figure S1 (see the Supporting Information, SI) The thin film size on the $\mathrm{MgO}(100)$ substrate was ca. $5 \times 10 \mathrm{~mm}$. The electrode was located at the edge of the film using Au paste, with a parallel electrode configuration. The distance between the electrodes is $4.0 \mathrm{~mm}$. The porous $\mathrm{Au}$ electrode is also covered at the side of the film. The conductivity of the $\mathrm{MgO}(100)$ substrate is negligible because its resistance is much higher than that of the thin film. The isotope effects were also investigated using humidified gases by $\mathrm{H}_{2} \mathrm{O}$ and $\mathrm{D}_{2} \mathrm{O}$.

For the measurement of the electromotive force (EMF) of an $\mathrm{H}_{2} /$ $\mathrm{O}_{2}$ cell, the sample was processed into pellets of $13 \mathrm{~mm} \phi$. Pt-loaded carbon paper sheets ( $\mathrm{Pt}: 1 \mathrm{mg} \mathrm{cm}{ }^{-2}$ ) were used as electrodes. The pelletized sample was sandwiched between an anode and cathode. Humidified hydrogen $(100 \mathrm{~mL} / \mathrm{min})$ and oxygen $(100 \mathrm{~mL} / \mathrm{min})$ were fed to the anode and cathode, respectively. The cell temperature and relative humidity were controlled to be $298 \mathrm{~K}$ and $70 \%$, respectively.

Infrared reflection absorption spectroscopy (RAS) was used to investigate the orientation of amide bonds for P-Asp film on sputtered Au film on $\mathrm{SiO}_{2}$ substrate. RAS measurement was performed with a 
FT-IR spectrometer equipped with a PR-510i accessory (FT/IR4200; JASCO). The polarized incident beam was reflected off the film surface at an incidence of $70^{\circ}$.

The p-MAIR measurements ${ }^{14-17}$ were performed on an FT-IR spectrometer (Nicolet 6700; Thermo Fisher Scientific) equipped with a mercury-cadmium-telluride (MCT) detector. Mirror-polished $\mathrm{MgO}(100)$ substrate was used for p-MAIR measurements. Singlebeam spectra were collected from $38^{\circ}$ to $8^{\circ}$ at $6^{\circ}$ steps in the range of the angle of incidence, as shown in Figure S2 of the SI. The aperture was fully opened (size of 150) and a metal plate with small pores was placed in the light path of the incident beam to prevent saturation. To obtain the $p$-polarized light, a $\mathrm{ZnSe}$ polarizer was used. The cleaned substrate was used as the reference data.

For measurements of the water adsorption isotherm per unit as a function of the $\mathrm{RH}$ for the thin film, the weight was obtained using a quartz crystal microbalance made by T.A. (the third author). The RH and temperature were controlled with a humidity-controlled and temperature-controlled chamber (SH-221; Espec Corp.).

\section{RESULTS AND DISCUSSION}

The electrical conductivity of the pelletized sample, which included sodium ions, was $4.3 \times 10^{-4} \mathrm{~S} \mathrm{~cm}^{-1}$ at $298 \mathrm{~K}$ and $70 \%$ $\mathrm{RH}$. It is reported that sodium ions may be coordinated by water molecules to conduct protons. ${ }^{18,19}$ In this case, the negative charge of a silicate layer is compensated by sodium cations that are octahedrally coordinated by water molecules in the interlayer space. It is suggested that protons are mobile in our P-Asp, similar to these reports. However, it is also possible that sodium cations are mobile. In order to determine whether the main charge carrier of this specimen is proton, the EMF of an $\mathrm{H}_{2} / \mathrm{O}_{2}$ cell of the pelletized sample was measured. The obtained EMF of the pelletized sample was ca. $0.9 \mathrm{~V} .{ }^{13}$ Here, we do not know about the mobility of the sodium ions, however, the EMF might be much lower if the sodium ions are also significantly mobile, which indicates that the proton transport number of P-Asp was high and that the main carrier was proton. The $\mathrm{H} / \mathrm{D}$ isotope effects using $\mathrm{H}_{2} \mathrm{O}$ and $\mathrm{D}_{2} \mathrm{O}$ as humidified gases were also investigated to determine the ionic transport carrier. Figure 1 shows impedance spectra under

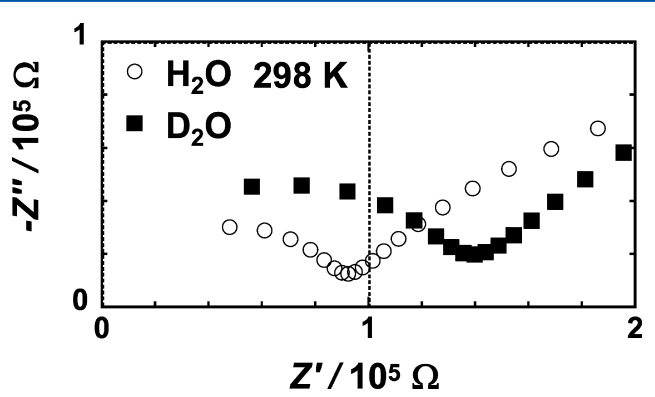

Figure 1. Impedance spectra under humidified conditions by $\mathrm{H}_{2} \mathrm{O}(\mathrm{O})$ and $\mathrm{D}_{2} \mathrm{O}(\boldsymbol{\square})$, respectively. The atmosphere around the sample was filled with the vapor of $\mathrm{H}_{2} \mathrm{O}$ or $\mathrm{D}_{2} \mathrm{O}$, where each vapor was generated by bubbling $\mathrm{H}_{2} \mathrm{O}\left(\mathrm{D}_{2} \mathrm{O}\right)$ with $\mathrm{N}_{2}$ gas.

humidified conditions by $\mathrm{H}_{2} \mathrm{O}$ and $\mathrm{D}_{2} \mathrm{O}$, respectively. The atmosphere around the sample was filled with the vapor of $\mathrm{H}_{2} \mathrm{O}$ or $\mathrm{D}_{2} \mathrm{O}$, where each vapor was generated by bubbling $\mathrm{H}_{2} \mathrm{O}$ $\left(\mathrm{D}_{2} \mathrm{O}\right)$ with $\mathrm{N}_{2}$ gas. When the surrounding atmosphere of the sample was changed from $\mathrm{D}_{2} \mathrm{O}$ to $\mathrm{H}_{2} \mathrm{O}$, the resistance decreased. Obvious difference in electrical conductivity was observed. The intersection of the semicircle corresponds to the resistance in Figure 1. The resistances under $\mathrm{H}_{2} \mathrm{O}$ and $\mathrm{D}_{2} \mathrm{O}$ vapor are 0.9 and $1.4 \times 10^{5} \Omega$, respectively. The resistance ratio is, therefore, very close to the theoretical value of the isotope effect ( $\sim 1.4$ for proton/deuteron). This result supports the EMF result and confirms that the main carrier is proton.

The proton conductivities of the pelletized and 60-nm thick film specimens of P-Asp at each relative humidity $(\mathrm{RH})$ are shown in Figure 2. The proton conductivity of the pelletized

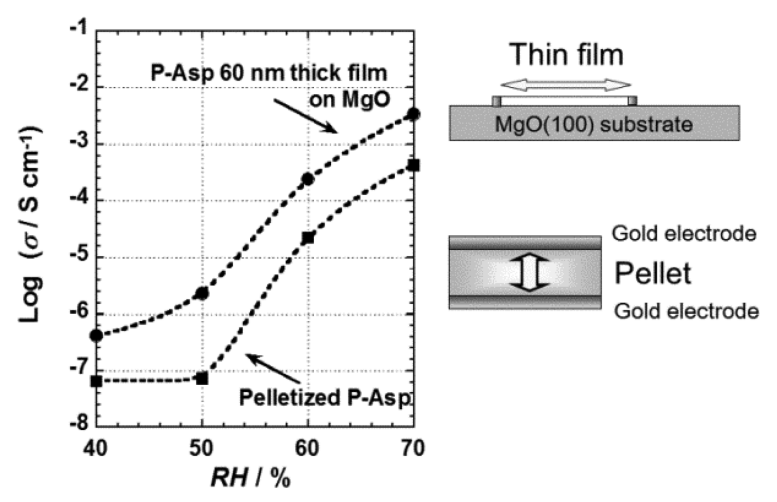

Figure 2. Relative humidity dependence of the proton conductivities

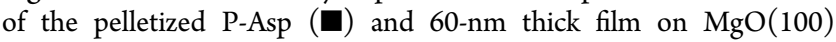
substrate $(O)$. The measurement temperature was $298 \mathrm{~K}$. Inset is electrode configuration for each measurement.

sample and 60-nm thick thin film logarithmically increased with $\mathrm{RH}$, which is a typical characteristic of proton conductive polymers. The proton conductivity of the thin film was $3.4 \times$ $10^{-3} \mathrm{~S} \mathrm{~cm}^{-1}$ under the $\mathrm{RH}$ of $70 \%$ at $298 \mathrm{~K}$, which was 1 order of magnitude higher than that of the pelletized samples. It is remarkable that the proton conductivity of this kind of polymer shows such a high value, in which the protons at the carboxylic acid groups serve as proton sources. ${ }^{20}$ It is also notable here that the conductivity enhancement was similarly observed in the thin film fabricated on amorphous $\mathrm{SiO}_{2}$ substrate, as we mentioned in the Introduction. ${ }^{13}$ As we have speculated, the enhancement may be attributed to the formation of a proton conducting path in the thin film. In our former report, we could not specify the origin of the enhancement. Therefore, we have performed further structural determination to investigate the molecular orientation in the thin film on the $\mathrm{MgO}$ single crystal, which is transparent in the IR region.

Infrared absorption spectroscopy is a powerful tool to obtain information about peptide structure, and polarized IR measurements enable one to analyze the orientation of functional group. Infrared reflection absorption spectroscopy (RAS), one of the polarized IR measurements, was used to investigate the orientation of peptide groups for P-Asp. In the reflection absorption (RA) spectrum shown in Figure 3(a), IR bands were observed at $1670,1620,1540,1410$, and $3300 \mathrm{~cm}^{-1}$. The RAS method detects the vibrational modes of which the transition dipole moment has a vector component perpendicular to the substrate. Therefore, the strong absorption band at $1670 \mathrm{~cm}^{-1}$ (the Amide I band, ${ }^{21,22}$ assigned to the $\mathrm{C}=\mathrm{O}$ stretching mode) in RA spectrum indicates that the transition dipole moment of the Amide I band directs perpendicular rather than parallel to the substrate surface.

The peak position of the Amide I band is related to the peptide main-chain structure. ${ }^{21,22}$ Judging from the observed peak position at $1670 \mathrm{~cm}^{-1}$, it is plausible that the P-Asp thin film exists in neither regular secondary structures such as $\alpha$ helix or $\beta$-sheet nor an irregular structure, because these secondary structures show the Amide I bands at the range of 


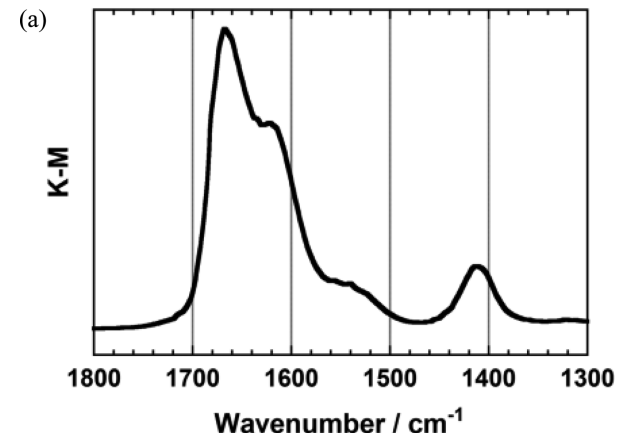

(b)

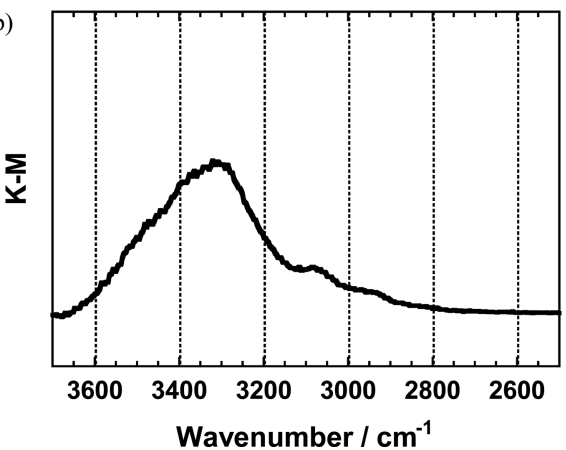

Figure 3. Infrared reflection absorption (RA) spectra (a) RA spectrum of P-Asp film in the wavenumber region of $1800-1300 \mathrm{~cm}^{-1}$. (b) RA spectrum in the wavenumber region of $3700-2500 \mathrm{~cm}^{-1}$. The vertical axis was converted by the Kubelka-Munk transformation.

$1640-1660,1620-1640$, and $1640-1660 \mathrm{~cm}^{-1}$, respectively. ${ }^{21,22}$ P-Asp would exist in other nonperiodic secondary structures, e.g., the $\beta$-turn ${ }^{21}$ or $\alpha$-sheet ${ }^{23}$ structures that give the IR absorption bands above $1660 \mathrm{~cm}^{-1}$. The RA bands also appear around 1620 and $1410 \mathrm{~cm}^{-1}$ in the RA spectrum (Figure 3(a)), and these bands are assignable to the antisymmetric and symmetric stretch modes, respectively, of the $\mathrm{COO}^{-}$group of the P-Asp side chain. ${ }^{24}$ Besides, an inhomogeneous broadening of the $\mathrm{N}-\mathrm{H}$ stretching band of the peptide main chain of P-Asp is seen at $3300 \mathrm{~cm}^{-1}$ due to the hydrogen bond formation of the $\mathrm{N}-\mathrm{H}$ groups of the peptide main chain (Figure 3(b)). The P-Asp main chain probably forms intramolecular or intermolecular hydrogen bonds in the thin film.

Infrared $p$-polarized multiple-angle incidence resolution ( $p$ MAIR) spectrometry was applied to the P-Asp film in order to determine the structure. ${ }^{16,17}$ Infrared p-MAIR spectrometry is being increasingly regarded as a powerful spectroscopic tool for revealing molecular orientation in thin films. Details of the measurement are given in the SI. Figure 4 shows in-plane (IP) and out-of-plane(OP) components of the p-MAIR spectra of the oriented P-Asp film sample. The IP and OP spectra are calculated from the experimental data using p-MAIR spectrometry analyzer software. The intensity of the $2 \times \mathrm{IP}$ signal is expected to be twice as large as the OP signal in the case spatial distribution of the transition moment vector of the vibrational mode is isotropic (see SI). The $2 \times$ IP signal becomes weaker when the transition moment vector directs the out-of-plane to the substrate, while the OP signal becomes weaker when the transition moment vector directs in-plane.

The signal intensity of the Amide I band at $1670 \mathrm{~cm}^{-1}$ is comparable between the $2 \times$ IP and OP spectra, and this result indicates that the transition moment vector of the amide I band at $1670 \mathrm{~cm}^{-1}$ directs perpendicular rather than parallel to the

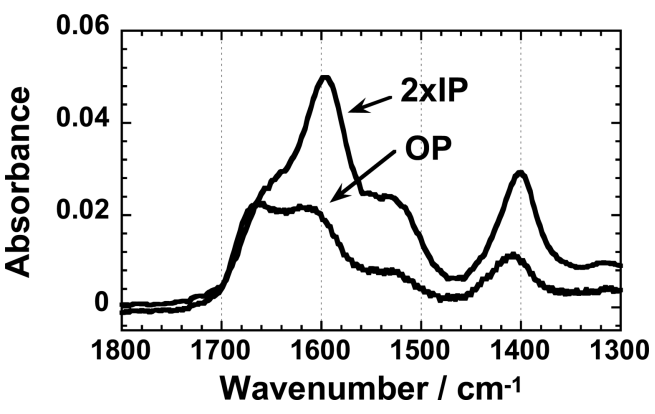

Figure 4. p-MAIR spectra of highly oriented P-Asp thin film. The IP component has two orthogonal directions of electric field. Therefore, the IP absorbance was multiplied by a factor of $2 .^{14}$.

substrate plane. This suggestion is consistent with the result of RAS. However, the symmetric and antisymmetric stretching bands of the $\mathrm{COO}^{-}$group at ca. 1400 and $1600 \mathrm{~cm}^{-1}$ gave the stronger signal in the $2 \times$ IP spectrum than in the doubled intensity of the OP spectrum. This result is explained by considering that the $\mathrm{O}-\mathrm{C}-\mathrm{O}$ plane of the $\mathrm{COO}^{-}$group of the Asp side chains lies parallel to the substrate plane. This model permits the in-plane orientation of the transition dipole moments of both symmetric and antisymmetric stretch motion of the $\mathrm{COO}^{-}$group. Since the result of RAS (Figure 3(a)) indicates an out-of-plane component of the transition dipole moment of the vibrations of the $\mathrm{COO}^{-}$group, we expect that not all of the side chains of P-Asp are completely oriented.

From the two polarized IR spectroscopy, the RAS and pMAIR spectrometry, we propose an $\alpha$-sheet-like model for the high proton conductivity in the thin film (Figure 5): high proton migration paths would be realized through $\alpha$-sheet layers, which percolate the in-plane formed by the stacked layer structure in the out-of-plane direction of the substrate. This model does not necessarily mean that the thin film is composed exclusively of $\alpha$-sheet layers. This model explains the peak position of the amide I band and the directionality of the peptide. This model for the high proton migration path is similar to the previous reports that ordered structures can improve the proton transport property. ${ }^{10,25,26}$ Tamura and Kawakami found that the composite bulk membrane containing uniaxially aligned sulfonated polyimide nanofibers exhibited a high proton conductivity. ${ }^{26}$ In this paper, the proposed structure in Figure 5 has been determined reasonably by the results of FT-IR measurements. However, it remains a hypothetical model without any further structural investigation to conclude the origin of enhanced conductivity. Additional analytical methods, such as grazing incidence X-ray diffraction under the humidified condition, are required to confirm the oriented thin film structure.

Another possible explanation for the proton conductivity enhancement results from space charge models. Enhancement phenomena of ionic conductivity in thin films (2D) of variety of ion conducting materials have been reported since more than 20 years. $^{27-39}$ For many types of inorganic ionic conductors, several theoretical models have been discussed, including space charge models, for understanding the enhancement phenomena of ionic conductivities. ${ }^{40-43}$ When the space charge at the interface is concerned, enhancement of ion conductivity depends on the thickness. As shown in the Figure 6, no clear-cut thickness dependence of proton conductivity for PAsp thin film for thicknesses of $20-60 \mathrm{~nm}$ was observed. This fact indicates that contribution of the space charge effect on the 


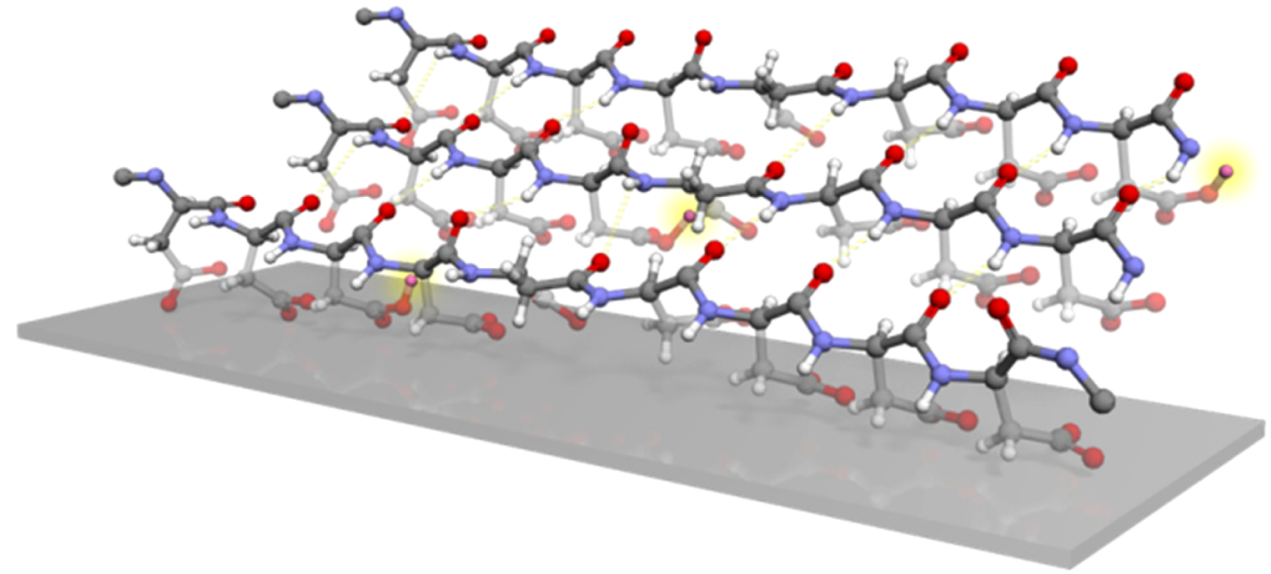

Figure 5. Proposed structure of the highly oriented P-Asp thin film. Pink atoms surrounded by yellow represent proton carriers of carboxylic acid groups. (C gray, $\mathrm{N}$ blue, $\mathrm{O}$ red, $\mathrm{H}$ white).

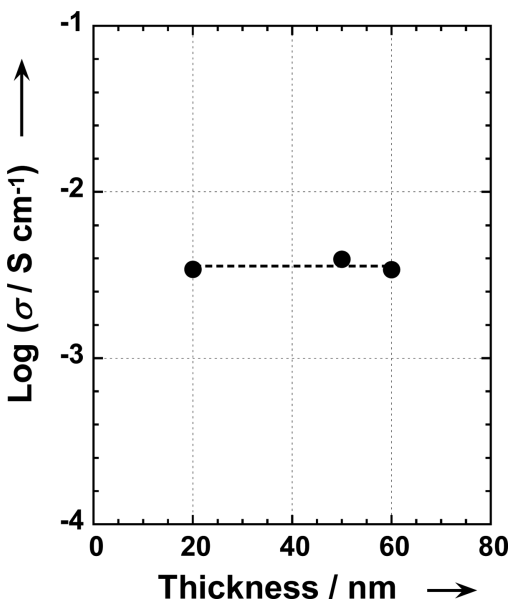

Figure 6. Thickness dependence of the proton conductivity for the PAsp thin film. Relative humidity was $70 \%$.

proton conductivity enhancement may be very small in these thin films.

Vogt et al. reported that swelling of water was enhanced as the film became thinner in the cases of some polymers. ${ }^{44}$ To determine the quantity of the water adsorption, a water adsorption isotherm was also measured. Figure 7 shows the water adsorption isotherm per unit as a function of the RH for the pelletized P-Asp and its thin film. The quantity of the water molecules in the thin film was measured using a quartz crystal microbalance. Their isotherm curves exhibited similar values at each RH. This fact showed that the amount of adsorbed water in the thin film and the pelletized sample are comparable with each other, therefore, the origin of proton conductivity enhancement was not in the difference of the amount of water adsorption in this study.

Finally, we show Arrhenius plots of the electrical conductivity in Figure 8. The activation energy $\left(E_{\mathrm{a}}\right)$ and pre-exponential factor $\left(\sigma_{0}\right)$ were obtained from the equation below.

$$
\sigma T=\sigma_{0} \exp \left(-\frac{E_{\mathrm{a}}}{k_{\mathrm{B}} T}\right)
$$

where $\sigma$ is the proton conductivity, $\sigma_{0}$ is the pre-exponential factor, $k_{\mathrm{B}}$ is the Boltzmann constant, and $T$ is the temperature. The activation energies for the proton conductivity were 0.34

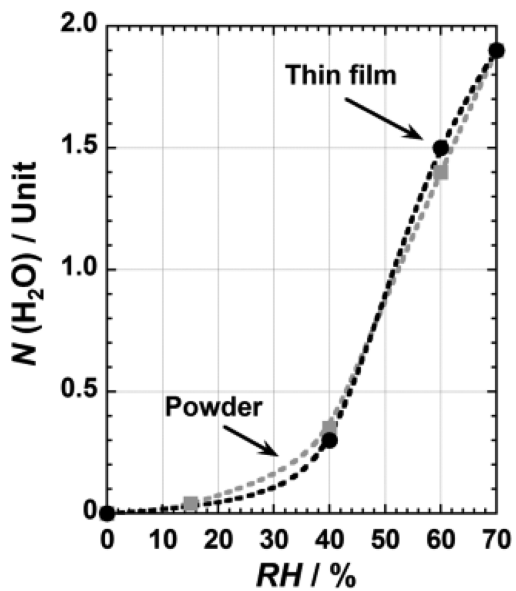

Figure 7. Water adsorption isotherm per unit as a function of the $\mathrm{RH}$ for the pelletized P-Asp and thin film. The measurement temperature was $298 \mathrm{~K}$.

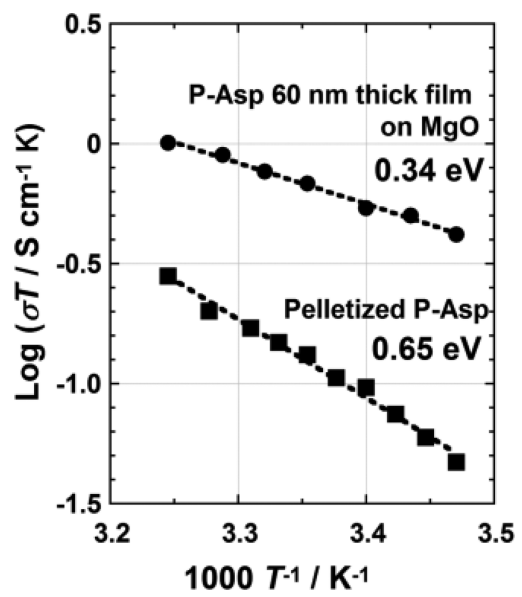

Figure 8. Temperature dependence of the proton conductivities of the pelletized P-Asp and 60-nm thick film on $\mathrm{MgO}(100)$ substrate. Relative humidity was $70 \%$.

$\mathrm{eV}$ for the thin film and $0.65 \mathrm{eV}$ for the pelletized sample, respectively. The activation energy of the thin film was half that of the pelletized sample. This value was the smallest among the thin films of P-Asp we have ever synthesized, including those in our previous reports. The low activation energy demonstrates 
that protons are more easily transported in thin film than in the pelletized sample. The polymer chain which is oriented in inplane direction may have caused the enhancement of in-plane proton conductivity in the thin film case. This fact is consistent with our proposed $\alpha$-sheet-like model that the more favorable proton conductive channels were created in the thin films. The conductivities at room temperature are comparable on both substrates, while activation energy of the thin film on $\mathrm{SiO}_{2}$ substrate is $0.53 \mathrm{eV},{ }^{13}$ which is about $0.2 \mathrm{eV}$ higher than the case of $\mathrm{MgO}$ substrate. It is reported that the $\mathrm{MgO}(100)$ surface is more positively charged than the $\mathrm{SiO}_{2}$ surface. ${ }^{45}$ This polar surface of the crystal plane may influence positively for the highly oriented structure of P-Asp using carboxylate groups at the side chains. Recently, we have found the substrate dependence between $\mathrm{MgO}$ and sapphire of the proton transport in the thin film and this was originated from the oriented structure at the interface. ${ }^{46}$ These results suggest to us a possible route to fabricate thin films of different proton transport properties by substrate selection. However, the preexponential factor $\left(\sigma_{0}\right)$ in the Arrhenius eq 1 for the $\mathrm{SiO}_{2}$ substrate is higher than that for the $\mathrm{MgO}$ substrate. It is known that the $\sigma_{0}$ term is related to the concentration of the conducting species. One possible reason for the different proton conductivity between the $\mathrm{SiO}_{2}$ and $\mathrm{MgO}$ substrates is derived from the different concentration of the mobile proton in the thin film. Another possibility is due to the different structure of the thin films between different substrates. Considering a significant difference of the activation energy for the proton conduction between the $\mathrm{SiO}_{2}$ and $\mathrm{MgO}$ substrates, we should carefully conclude the origin for the difference of the pre-exponential factor. To reveal the origin of the difference, the solid state NMR measurements will be a useful tool to reveal the proton mobility.

In order to check the molecular orientation in the pelletized sample, attenuated total reflection (ATR) measurements were carried out with an FT-IR spectrometer (Nicolet 6700; Thermo Fisher Scientific). Figure 9 shows the ATR spectra of

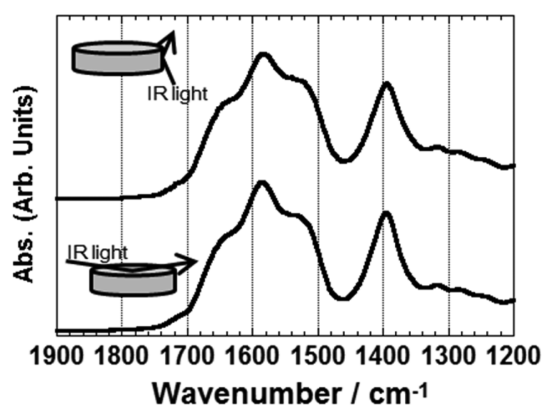

Figure 9. ATR spectra of the pelletized P-Asp. Inset shows the configuration between the pelletized sample direction and IR light direction.

the pelletized sample in each configuration. Inset shows the configuration between the pelletized sample direction and IR light direction. These spectra are identical. This result suggests a random orientation in the pelletized sample. The Amide I band correspondingly appears at ca. $1650 \mathrm{~cm}^{-1}$ in the ATR result, consistent with the picture that the peptide main chain of P-Asp exists in the irregular structure that gives the band at $1640-1660 \mathrm{~cm}^{-1}$. $^{21,22}$ There is no scaffold for inducing the oriented structure, which may result in a random orientation of P-Asp. Such a difference in structure was suggested to be the origin of the remarkable difference in proton conductivity. Consideration of these experimental facts will lead to the conclusion that the proton conductivity enhancement in the $60-\mathrm{nm}$ thick film of P-Asp results from the highly oriented structure in the thin film.

\section{CONCLUSIONS}

A remarkable enhancement of proton conductivity in polypeptide thin films has been achieved, which is attributable to the highly oriented structure on the $\mathrm{MgO}(100)$ substrate. We found that the P-Asp thin film prepared on $\mathrm{MgO}(100)$ substrate showed one order of magnitude higher proton conductivity and two times smaller activation energy than that of their bulk sample. This study has proposed a new route to produce highly proton-conductive materials using the concept of an oriented thin film structure without any strong acid groups and shows great potential for application in the field of solid state ionics.

\section{ASSOCIATED CONTENT}

\section{Supporting Information}

Details in impedance plots and $p$-polarized multiple-angle incidence resolution spectrometry ( $\mathrm{p}$-MAIRS). This material is available free of charge via the Internet at http://pubs.acs.org.

\section{AUTHOR INFORMATION}

\section{Corresponding Author}

*Phone: +81-761-51-1541; e-mail: ynagao@jaist.ac.jp.

\section{Present Address}

$\checkmark$ J.M.: Faculty of Science, Yamagata University, Kojirakawamachi 1-4-12, Yamagata 990-560, Japan.

\section{Notes}

The authors declare no competing financial interest.

\section{ACKNOWLEDGMENTS}

This work was supported by the Japan Society for the Promotion of Science (JSPS) through the Funding Program (GR060) for Next Generation World-Leading Researchers (NEXT Program), initiated by the Council for Science and Technology Policy (CSTP).

\section{REFERENCES}

(1) Rikukawa, M.; Sanui, K. Proton-conducting polymer electrolyte membranes based on hydrocarbon polymers. Prog. Polym. Sci. 2000, $25,1463-1502$

(2) Kerres, J. A. Development of ionomer membranes for fuel cells. J. Membr. Sci. 2001, 185, 3-27.

(3) Allcock, H. R.; Wood, R. M. Design and synthesis of ionconductive polyphosphazenes for fuel cell applications: Review. $J$. Polym. Sci., Part B 2006, 44, 2358-2368.

(4) Jagur-Grodzinski, J. Polymeric materials for fuel cells: Concise review of recent studies. Polym. Adv. Technol. 2007, 18, 785-799.

(5) Laberty-Robert, C.; Valle, K.; Pereira, F.; Sanchez, C. Design and properties of functional hybrid organic-inorganic membranes for fuel cells. Chem. Soc. Rev. 2011, 40, 961-1005.

(6) Miyatake, K.; Bae, B.; Watanabe, M. Fluorene-containing cardo polymers as ion conductive membranes for fuel cells. Polym. Chem. 2011, 2, 1919-1929.

(7) Hsu, W. Y.; Gierke, T. D. Ion transport and clustering in nafion perfluorinated membranes. J. Membr. Sci. 1983, 13, 307-326.

(8) Kreuer, K. D. On the development of proton conducting polymer membranes for hydrogen and methanol fuel cells. J. Membr. Sci. 2001, $185,29-39$. 
(9) Mauritz, K. A.; Moore, R. B. State of understanding of Nafion. Chem. Rev. 2004, 104, 4535-4585.

(10) Schmidt-Rohr, K.; Chen, Q. Parallel cylindrical water nanochannels in Nafion fuel-cell membranes. Nat. Mater. 2008, 7 (1), 7583.

(11) Nagao, Y. Proton transport property of Nafion thin films on $\mathrm{MgO}(100)$ with anisotropic molecular structure. e-J. Surf. Sci. Nanotechnol. 2012, 10, 114-116.

(12) Nagao, Y. Highly oriented sulfonic acid groups in a Nafion thin film on Si substrate. J. Phys. Chem. C 2013, 117, 3294-3297.

(13) Nagao, Y.; Iguchi, F.; Sata, N.; Yugami, H. Synthesis and proton transport property of poly(aspartic acid) thin film on $\mathrm{SiO}_{2}$ substrate. Solid State Ionics 2010, 181, 206-209.

(14) Hasegawa, T. A novel measurement technique of pure out-ofplane vibrational modes in thin films on a nonmetallic material with no polarizer. J. Phys. Chem. B 2002, 106, 4112-4115.

(15) Hasegawa, T.; Matsumoto, L.; Kitamura, S.; Amino, S.; Katada, S.; Nishijo, J. Optimum condition of Fourier transform infrared multiple-angle incidence resolution spectrometry for surface analysis. Anal. Chem. 2002, 74, 6049-6054.

(16) Hasegawa, T. Advanced multiple-angle incidence resolution spectrometry for thin-layer analysis on a low-refractive-index substrate. Anal. Chem. 2007, 79, 4385-4389.

(17) Hasegawa, T.; Itoh, Y.; Kasuya, A. Experimental optimization of p-polarized MAIR spectrometry performed on a fourier transform infrared spectrometer. Anal. Sci. 2008, 24, 105-109.

(18) Borowski, M.; Wolf, I.; Gies, H. Investigation of proton dynamics within the hydrogen-bond network of the layer silicate NaRUB-18. Chem. Mater. 2002, 14, 38-43.

(19) Ishimaru, S.; Togawa, M.; Ikeda, R.; Shimizu, T.; Shinohara, E.; Umemura, Y. Complex impedance and solid-state NMR studies on a novel high proton conductor Na-RUB-18. Bull. Chem. Soc. Jpn. 2006, 79, 656-659.

(20) Liu, B.; Hu, W.; Robertson, G. P.; Guiver, M. D. Poly(aryl ether ketone)s with carboxylic acid groups: Synthesis, sulfonation and crosslinking. J. Mater. Chem. 2008, 18, 4675-4682.

(21) Krimm, S.; Bandekar, J. Vibrational spectroscopy and conformation of peptides, polypeptides, and proteins. Adv. Protein Chem. 1986, 38, 181-364.

(22) Dong, A.; Huang, P.; Caughey, W. S. Protein secondary structures in water from 2nd-derivative amide-I infrared-spectra. Biochemistry 1990, 29, 3303-3308.

(23) Torii, H. Amide I infrared spectral features characteristic of some untypical conformations appearing in the structures suggested for amyloids. J. Phys. Chem. B 2008, 112, 8737-8743.

(24) Barth, A.; Zscherp, C. What vibrations tell us about proteins. Q. Rev. Biophys. 2002, 35, 369-430.

(25) Dong, B.; Gwee, L.; Salas-de, 1. C. D.; Winey, K. I.; Elabd, Y. A. Super proton conductive high-purity Nafion nanofibers. Nano Lett. 2010, 10, 3785-3790.

(26) Tamura, T.; Kawakami, H. Aligned electrospun nanofiber composite membranes for fuel cell electrolytes. Nano Lett. 2010, 10, $1324-1328$.

(27) Schreck, E.; Laeuger, K.; Dransfeld, K. Enhanced ionic conductivity at the interface between sapphire and solid lithium iodide. Z. Phys. B: Condens. Matter 1986, 62, 331-334.

(28) Muehlherr, S.; Laeuger, K.; Schreck, E.; Dransfeld, K.; Nicoloso, $\mathrm{N}$. The ionic conductivity profile of thin evaporated silver chloride films on a planar sapphire substrate. Solid State Ionics 1988, 28-30, $1495-1505$.

(29) Modine, F. A.; Lubben, D.; Bates, J. B. Electrical conduction in calcium fluoride and $\mathrm{CaF}_{2}-\mathrm{Al}_{2} \mathrm{O}_{3}$ nanocomposite films on alumina substrates. J. Appl. Phys. 1993, 74, 2658-2664.

(30) Lubben, D.; Modine, F. A. Enhanced ionic conduction mechanisms at $\mathrm{LiI} / \mathrm{Al}_{2} \mathrm{O}_{3}$ interfaces. J. Appl. Phys. 1996, 80, 51505157.

(31) Kosacki, I.; Anderson, H. U. Nanostructured oxide thin films for gas sensors. Sens. Actuators, B 1998, B48, 263-269.
(32) Kosacki, I.; Suzuki, T.; Petrovsky, V.; Anderson, H. U. Electrical conductivity of nanocrystalline ceria and zirconia thin films. Solid State Ionics 2000, 136-137, 1225-1233.

(33) Sata, N.; Eberman, K.; Eberl, K.; Maier, J. Mesoscopic fast ion conduction in nanometre-scale planar heterostructures. Nature 2000, 408, 946-949.

(34) Guo, X. X.; Maier, J. Ionic conductivity of epitactic MBE-grown $\mathrm{BaF}_{2}$ films. Surf. Sci. 2004, 549, 211-216.

(35) Kosacki, I.; Rouleau, C. M.; Becher, P. F.; Bentley, J.; Lowndes, D. H. Nanoscale effects on the ionic conductivity in highly textured YSZ thin films. Solid State Ionics 2005, 176, 1319-1326.

(36) Azad, S.; Marina, O. A.; Wang, C. M.; Saraf, L.; Shutthanandan, V.; McCready, D. E.; El-Azab, A.; Jaffe, J. E.; Engelhard, M. H.; Peden, C. H. F.; Thevuthasan, S. Nanoscale effects on ion conductance of layer-by-layer structures of gadolinia-doped ceria and zirconia. Appl. Phys. Lett. 2005, 86, 131906/1-131906/3.

(37) Furusawa, S.-i.; Tabuchi, H.; Tsurui, T. Ionic conductivity of lithium alumino-silicate thin films on $\mathrm{SiO}_{2}$ glass and $\mathrm{Al}_{2} \mathrm{O}_{3}$ substrates. Solid State Ionics 2007, 178, 1033-1038.

(38) Peters, A.; Korte, C.; Hesse, D.; Zakharov, N.; Janek, J. Ionic conductivity and activation energy for oxygen ion transport in superlattices-The multilayer system $\mathrm{CSZ}\left(\mathrm{ZrO}_{2}+\mathrm{CaO}\right) / \mathrm{Al}_{2} \mathrm{O}_{3}$. Solid State Ionics 2007, 178, 67-76.

(39) Furusawa, S.-i.; Kamiyama, A.; Tsurui, T. Fabrication and ionic conductivity of amorphous lithium meta-silicate thin film. Solid State Ionics 2008, 179, 536-542.

(40) Maier, J. Ionic conduction in space charge regions. Prog. Solid State Chem. 1995, 23, 171-263.

(41) Agrawal, R. C.; Gupta, R. K. Superionic solids: Composite electrolyte phase-An overview. J. Mater. Sci. 1999, 34, 1131-1162.

(42) Schoonman, J. Nanoionics. Solid State Ionics 2003, 157, 319326.

(43) Maier, J. Nanoionics: Ion transport and electrochemical storage in confined systems. Nat. Mater. 2005, 4, 805-815.

(44) Vogt, B. D.; Soles, C. L.; Jones, R. L.; Wang, C.-Y.; Lin, E. K.; Wu, W.-1.; Satija, S. K.; Goldfarb, D. L.; Angelopoulos, M. Interfacial effects on moisture absorption in thin polymer films. Langmuir 2004, 20, 5285-5290.

(45) Oka, K.; Furusawa, K. In Electrical Phenomena at Interfaces: Fundamentals: Measurements, and Applications, 2nd ed.; Ohshima, H., Furusawa, K., Eds.; Surfactant Science Series; Marcel Dekker, Inc.: New York, 1998; Vol. 76, pp 198.

(46) Nagao, Y. Substrate dependence of the proton transport and oriented structure in oligo[(1,2propanediamine)-alt-(oxalic acid)] thin films. Chem. Lett. 2013, 42, 468-470. 